\title{
Concurrent pelvic radiation with weekly low-dose cisplatin and gemcitabine as primary treatment of locally advanced cervical cancer: A phase II study
}

\author{
Hend Ahmed EL-Hadaad ${ }^{1}$, Hanan Ahmed Wahba ${ }^{1}$, Sameh Roshdy ${ }^{2}$, Anas Gamal ${ }^{3}$ \\ ${ }^{1}$ Department of Clinical Oncology \& Nuclear Medicine, Mansoura University, Mansoura, Egypt \\ ${ }^{2}$ Department of Surgical Oncology, Mansoura University, Mansoura, Egypt \\ ${ }^{3}$ Department of Gynecology and Obstetrics, Mansoura University, Mansoura, Egypt
}

Received March 29, 2015; Revised May 24, 2015; Accepted May 29, 2015; Published Online July 14, 2015

\section{Original Article}

\begin{abstract}
Purpose: This study was done to evaluate response, compliance and survival of weekly low dose cisplatin $\left(20 \mathrm{mg} / \mathrm{m}^{2}\right)$ and gemcitabine $\left(125 \mathrm{mg} / \mathrm{m}^{2}\right)$ concurrently with pelvic radiation as primary treatment of stage IIB-IIIB cervical cancer. Methods: External radiation consisted of $50 \mathrm{~Gy} / 25$ fractions using 6-10 MV photon followed by $600 \mathrm{cGy}$ boost to parametrium if it was still felt thickened. Then, intracavitary radiotherapy to deliver $60 \mathrm{~Gy}$ at point A. Chemotherapy consisted of gemcitabine at a dose of 125 $\mathrm{mg} / \mathrm{m}^{2}$ was given by i.v infusion over 30 minutes immediately after cisplatin $20 \mathrm{mg} / \mathrm{m}^{2}$ weekly for 5 weeks during EBRT. Fortyfive eligible patients received the treatment protocol. Results: Toxicity was tolerable and manageable. No grade 4 toxicity while grade 3 was recorded in hematologic one only. In order of frequency; diarrhea, nausea and vomiting, and anemia $(50 \%, 40 \%$, $35.5 \%$ ) were most common adverse events. Overall clinical response rate was $93.4 \%$ with pathological complete response of $62.2 \%$. After median follow-up of 20 months, 2-year survival and progression-free survival rates were $90.5 \%$ and $81 \%$ respectively. Conclusion: Weekly combination of low- dose cisplatin and gemcitabine given concurrently with pelvic radiotherapy in primary treatment of locally advanced cervical cancer resulted in a high response rate with a good compliance. Further exploration is needed for the use of this approach prior to incorporating it into routine clinical care through phase III clinical trial.
\end{abstract}

Keywords: Cervical Carcinoma; Gemcitabine; Chemo-Radiotherapy; Cisplatin

\section{Introduction}

Uterine cervical cancer is the second most common female malignancy in the world. ${ }^{1}$ However, the incidence of it has decreased markedly in recent decades following the introduction of screening programs. ${ }^{2}$ Usually cervical carcinoma presents as a locally advanced disease with parametrial infiltration in about half of the women especially in developing countries due to lack of early detection programs. Co-chrane meta-analysis in nearly 5000 patients strongly suggested that chemo-radiotherapy leads to greater disease-free and overall survival rates and better local control than with radical radiotherapy alone in locally advanced cervical cancer. ${ }^{3}$ Induction chemo-radiotherapy can be followed by surgical consolidation. This approach has a sound theoretical bases as surgery may eliminate residual disease which otherwise could be resistant to chemo-radiation.

Gemcitabine has shown promising results in some phase I and II trials as a radiosensitizing agent. ${ }^{4-6}$ Gemcitabine is activated intracellulary by deoxycytidine kinase and is converted into two active metabolites gemcitabine diphosphate and tri- phosphate which target DNA and RNA. It is considered to be an attractive compound to combine with ionizing radiation for several reasons: 1) It may inhibit repair of the DNA damage caused by radiation leading to increased cell death; 2) It may induce cell redistribution causing cells to accumulate in more radiosensitive phase of cell cycle; 3) Increased the radiosensitvity of hypoxic cells due to tumor shrinkage. Mc Cormach et al. ${ }^{7}$ used gemcitabine with radiation in patients with locally advanced cervical cancer and concluded that gemcitabine is more potent radiosensitier than cisplatin while Srivastava et al. ${ }^{8}$ found that cisplatin appeared to be better than gemcitabine when used as radiosensitizer.

This study was undertaken to evaluate the efficacy and safety of a concurrent regimen of gemcitabine / cisplatin and radiotherapy in women with stage IIB-IIIB cervical carcinoma.

\section{Methods and Materials}

Women with untreated invasive squamous cell carcinoma of the cervix of FIGO stage IIB-IIIB were enrolled in this study 
from April 2010 to December 2013. All cases were confirmed histologically. Each patient was required to undergo a complete physical examination, pelvic examination, chest radiography, intravenous pylography (IVP) and abdomino-pelvic magnetic resonance imaging (MRI). Sigmoidoscopy and cystoscopy were performed if needed. All patients were to have ECOG performance status score of $0-2$, adequate bone marrow reserve (ANC $>1500 / \mathrm{mm}^{3}$, platelet count $>100.000 /$ $\mathrm{mm}^{3}$ and $\mathrm{Hb} \geq 10 \mathrm{~g} / \mathrm{dL}$ ), renal function (serum creatinine $\leq 1.5$ $\mathrm{mg} / \mathrm{dl}$ ), liver function (bilirubin $<2$ times upper limit of normal (ULN) and SGOT<3 times ULN). Patients with diseases outside the pelvis or with para-aortic lymph node were excluded from the study.

\section{Radiotherapy}

External beam radiotherapy (EBRT), 50 Gy/25 fractions was delivered using 6-10 MV photon beam through 4 field box technique. Typical field borders were; upper border was at L4-5 inter space; lower border at lower most part of obturator foramen and was modified according to vaginal extent of the disease; anterior border was at the anterior symphysis pubis and the posterior border at S2-S3 junction. Additional 600 cGy may be boosted to the invaded parametrium if was still felt thickened on evaluation by pelvic examination.

For intracavitary radiotherapy (ICRT), patients were referred to other centers after completion of EBRT. All patients received single application of ICRT to deliver $20 \mathrm{~Gy}$ at point A.

\section{Chemotherapy}

Gemcitabine at a dose of $125 \mathrm{mg} / \mathrm{m}^{2}$ was given by i.v infusion over 30 minutes in $300 \mathrm{ml}$ normal saline immediately after cisplatin $20 \mathrm{mg} / \mathrm{m}^{2}$ weekly for 5 weeks during EBRT, beginning on first day of radiation. Cisplatin infusion was administered with adequate pre and post hydration; started $12 \mathrm{~h}$ before infusion. Prophylactic antiemetic (dexamethasone, ranitidine and ondansetron) were given before cisplatin.

Chemotherapy and radiotherapy toxicity were assessed according to Common Terminology Criteria for Adverse Events V3 ${ }^{9}$ and RTOG classification ${ }^{10}$ respectively.

When hematological toxicity was $\geq$ grade 3 , chemotherapy and radiotherapy were withheld. For grade 2 hematological toxicity only chemotherapy was withheld. When non hematological acute radiation morbidity was $\geq$ grade 3 , radiation was interrupted.

Response to treatment was assessed according to response evaluation criteria in solid tumors (RECIST) ${ }^{11}$ after 3 weeks of end of treatment.

Surgery was scheduled within 4-6 weeks after completion of chemoradiotherapy for patients who became operable. Histopathological response to treatment was defined as complete regression (pCR) with no residual tumor cells and subtotal regression (PSR) with $<10 \%$ viable tumor cells.

The end points of this study were response rate, tolerability, overall survival (OAS) and progression free survival (PFS) rates. The OAS was calculated from date of start treatment to date of death or lost follow-up while PFS was calculated from date of end of treatment to date of documented progression. Patients were assessed every 3 months for first year then every 6 months thereafter by clinical examination and abdomino pelvic MRI.

Statistical analysis: SPSS version15.0 (Chicago, IL, USA) was used. Data expressed as Number and percentile. OAS and PFS assessed by using Kaplan-Meier.

\section{Results}

A total of 45 patients were treated on protocol. Pre-treatment clinical characteristics are summarized in Table 1. Median age was 44 years. About $49 \%$ of patients had ECOGPS of 0 . Most patients had stage IIB (60\%). Moderately differentiated squamous cell carcinoma was found in $49 \%$ while ulcerative pathology was reported in $64.5 \%$.

TABLE 1: Patients characterestics $(\mathrm{n}=45)$.

\begin{tabular}{|c|c|}
\hline Character & $\mathrm{N}(\%)$ \\
\hline Age & \\
\hline Median (range) & $44(27-68)$ \\
\hline ECOGPS & \\
\hline 0 & $22(48.9)$ \\
\hline 1 & $18(40)$ \\
\hline 2 & $5(11.1)$ \\
\hline FIGO Stage & \\
\hline IIB & $27(60)$ \\
\hline IIIA & $12(26.7)$ \\
\hline IIIB & $6(13.3)$ \\
\hline Grade & \\
\hline Well differentiated & $16(35.5)$ \\
\hline Moderately differentiated & $22(48.9)$ \\
\hline Poorly differentiated & 7(15.6) \\
\hline Gross pathology & \\
\hline Ulcerative & $29(64.5)$ \\
\hline Exophytic & $10(22.2)$ \\
\hline Infiltrative & $6(13.3)$ \\
\hline
\end{tabular}

TABLE 2: Acute toxicities.

\begin{tabular}{|c|c|c|c|c|c|c|c|c|}
\hline \multirow[t]{3}{*}{ Toxicity } & \multicolumn{8}{|c|}{ Grade } \\
\hline & \multicolumn{2}{|c|}{1} & \multicolumn{2}{|c|}{2} & \multicolumn{2}{|c|}{3} & \multicolumn{2}{|c|}{4} \\
\hline & $\mathrm{N}$ & $\%$ & $\mathrm{~N}$ & $\%$ & $\mathrm{~N}$ & $\%$ & $\mathrm{~N}$ & $\%$ \\
\hline Neutropenia & 4 & 8.9 & 6 & 13.3 & 2 & 4.4 & 0 & 0 \\
\hline Thrombocytopenia & 6 & 13.3 & 7 & 15.6 & 1 & 2.2 & 0 & 0 \\
\hline Anemia & 8 & 17.8 & 5 & 11.1 & 3 & 6.6 & 0 & 0 \\
\hline Nausea/vomiting & 10 & 22.2 & 8 & 17.8 & 0 & 0 & 0 & 0 \\
\hline Dermatitis & 5 & 11.1 & 3 & 6.6 & 0 & 0 & 0 & 0 \\
\hline Proctitis & 7 & 15.6 & 5 & 11.1 & 0 & 0 & 0 & 0 \\
\hline Cystitis & 6 & 13.3 & 8 & 17.8 & 0 & 0 & 0 & 0 \\
\hline Diarrhea & 11 & 24.4 & 12 & 26.7 & 0 & 0 & 0 & 0 \\
\hline
\end{tabular}


TABLE 3: Response rate.

\begin{tabular}{l|l|l}
\hline \hline Response & N & $\%$ \\
\hline Complete response (CR) & 34 & 75.6 \\
Partial response (PR) & 8 & 17.8 \\
Stable disease (SD) & 2 & 4.4 \\
Progressive disease (PD) & 1 & 2.2 \\
Pathological complete response (pCR) & 28 & 62.2 \\
\hline \hline
\end{tabular}

All patients completed the treatment protocol with interruption in 3 patients only $(6.6 \%)$ of about 3-6 days. Toxicity was moderate. The incidence and severity of acute toxicities are shown in Table 2 . No grade 4 toxicity was recorded while grade 3 was found in hematologic one only. Fortunately, neither reported ototoxicity nor nephrotoxicity. Among hematologic adverse events; anemia was the most common (35.5\%) followed by thrombocytopenia (31\%). Diarrhea, nausea and vomiting, and cystitis were found in respect to order of frequency as following: 51\%, 40\%, 31\%.

Overall clinical response rate was $93.4 \%$; stable and progressive diseases were $4.4 \%$ and $2.2 \%$ respectively. Operable patients underwent type III radical hysterectomy with bilateral pelvic lymphadenectomy and pathological complete response was $62.2 \%$ (Table 3 ).

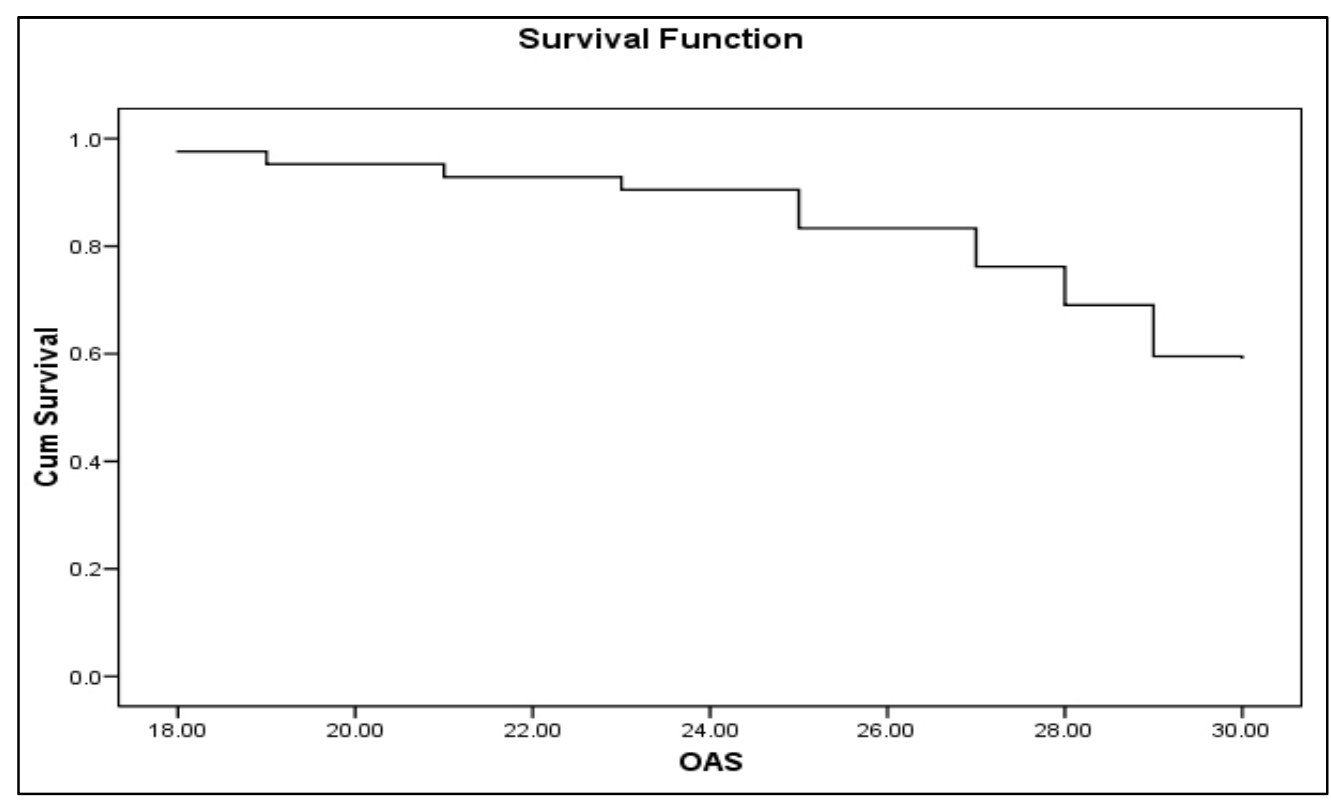

FIG. 1: Overall survival of all cases.

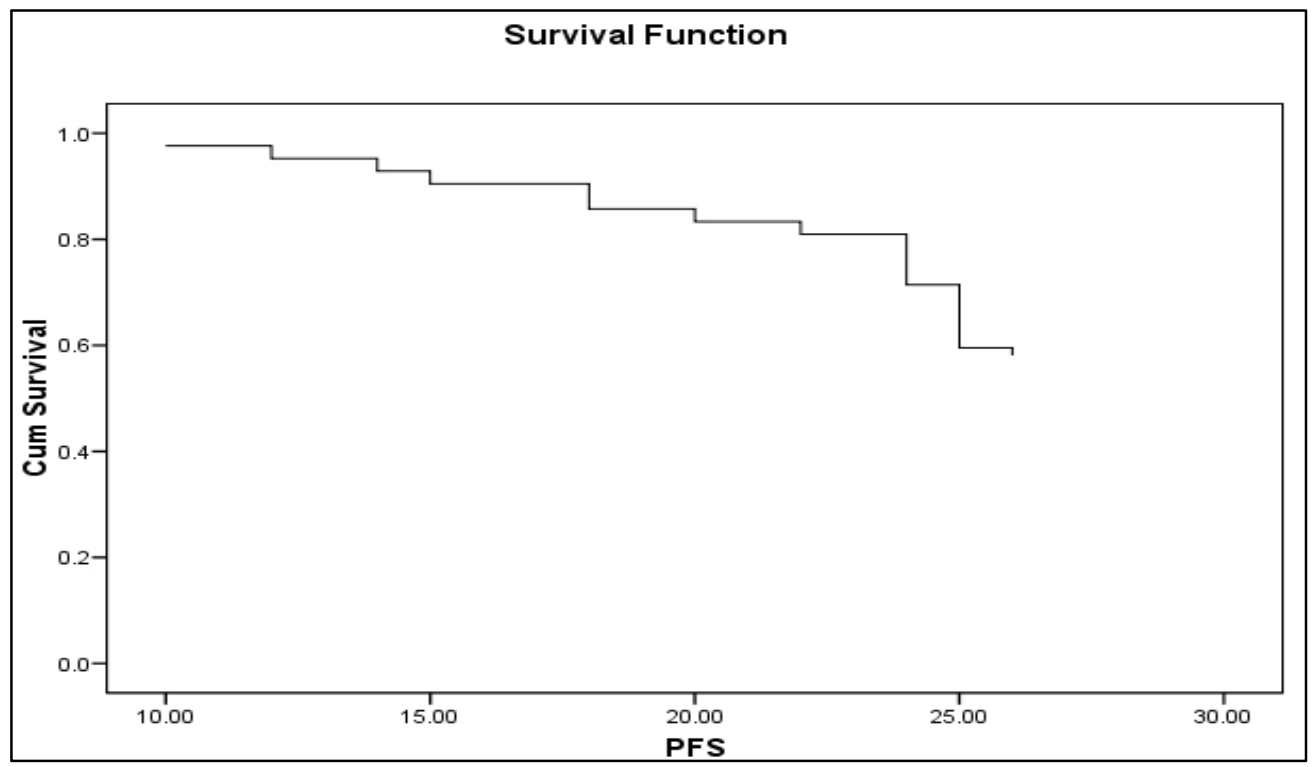

FIG. 2: Progression free survival. 
During follow-up period, two patients died of unrelated causes of diseases; so survival rates were assessed in $43 \mathrm{pa}-$ tients. Figure 1 and Figure 2 showed 2-year overall survival (OAS) and progression -free survival (PFS) rates $(90.5 \%$ and $81 \%$ ) respectively. After median follow-up of 20 months; median survival time was 30 months while that of PFS was 26 months.

\section{Discussion}

Treatment of carcinoma of the cervix has evolved immensely over the last decade. For locally advanced carcinoma of the cervix, overall prognosis has always been somber until the shift in the treatment paradigm came with concurrent chemoradiotherapy protocol. The most standard approach is the use of concurrent cisplatin $40 \mathrm{mg} / \mathrm{m}^{2}$ weekly along with radiation but locoregional failure range from $30 \%$ to $40 \% .^{12,13}$ Gemcitabine is a cell cycle specific cytotoxic agent that has shown antitumor activity against a variety of solid tumors e.g. lung, pancreas, breast and bladder.

Hernandez et al. ${ }^{14}$ have demonstrated the radiosensitizing effect of gemcitabine against cervical cancer cell line. Overall toxicity was acceptable and tolerable. No patients demonstrated ototoxicity or nephrotoxicity in this study. However; previous study using weekly cisplatin $40 \mathrm{mg} / \mathrm{m}^{2}$ had reported ototoxicity in $10 \%{ }^{15}$ while nephrotoxicity occurred in $42.9 \%$ in a study using weekly combination of gemcitabine with this standard dose of cisplatin. ${ }^{16}$

Among hematologic toxicity; anemia was the most common (35.5\%) followed by thrombocytopenia (31\%) then neutropenia $(26.6 \%)$ with grade 3 in $4.4 \%$ and no grade 4 that comparable to finding by Umanzor et al. ${ }^{6}$ However; Zarba et al. ${ }^{5}$ recorded grade 4 neutropenia in $4 \%$. Anemia was found in $50 \%$ of patients treated with gemcitabine $125 \mathrm{mg} / \mathrm{m}^{2}$ and cisplatin $40 \mathrm{mg} / \mathrm{m}^{2}{ }^{4}$ In our study; diarrhea, nausea and vomiting were the most common non hematologic adverse events (51\% and $40 \%$ respectively) with no grade 3 nor 4 toxicity while Khalil et al. ${ }^{17}$ reported grade 3 and 4 diarrhea in $36.6 \%$ when used cisplatin $40 \mathrm{mg} / \mathrm{m}^{2}$ concurrent with radiotherapy. Grade 1 and 2 diarrhea was observed in $50 \%$ of patients treated with gemcitabine $300 \mathrm{mg} / \mathrm{m}^{2}{ }^{4}$ and 2-year survival rate of $63 \%$ while that in our study was $94 \%$ but their patients were of stage IB2-IVA. Overall clinical response rate in our study was 93.4\%, 2-year OAS and PFS rates were $90.5 \%$ and $81 \%$ respectively. In a study conducted by Pattaranutaporn et $a l^{4}$ response rate was comparable to ours. Also, Zarba et al. ${ }^{5}$ had comparable response rate but with higher toxicity may be due to higher dose of cisplatin. Overall response rate was higher than that using cisplatin only $40 \mathrm{mg} / \mathrm{m}^{2}$ (79.6\%).

\section{Conclusion}

Weekly combination of low- dose cisplatin and gemcitabine given concurrently with pelvic radiotherapy in primary treatment of locally advanced cervical cancer resulted in a high response rate with a good compliance. Further exploration is needed for the use of this approach prior to incorporating it into routine clinical care through phase III clinical trial.

\section{Conflict of interest}

The authors declare that they have no conflicts of interest. The authors alone are responsible for the content and writing of the paper.

\section{References}

1. Ferlay J, Bray F, Pisani P, et al. Globocan 2002: Cancer Incidence, Mortality and Prevelance Worldwide. IARC Cancer Base No. 5, version 2.0.Lyon: IARC Press, 2004.

2. Park JH, Kim YS, Ahn SD, et al. Concurrent chemoradiotherapy or radiotherapy alone for locally advanced cervical cancer in elderly women. Tumori. 2010;96:959-65.

3. Green J, Kirwan J, Tierney J, et al. Concomitant chemotherapy and radiation therapy for cancer of the uterine cervix. Cochrane Database Syst Rev. 2005;CD002225.

4. Pattaranutaporn P, Thirapakawong C, Chansilpa Y, et al. Phase II study of concurrent gemcitabine and radiotherapy in locally advanced stage IIIB cervical carcinoma. Gynecol Oncol. 2001;81:404-7.

5. Zarbá JJ, Jaremtchuk AV, Gonzalez Jazey P, et al. A phase I-II study of weekly cisplatin and gemcitabine with concurrent radiotherapy in locally advanced cervical carcinoma. Ann Oncol. 2003;14:1285-90.

6. Umanzor J, Aguiluz M, Pineda C, et al. Concurrent cisplatin/gemcitabine chemotherapy along with radiotherapy in locally advanced cervical carcinoma: a phase II trial. Gynecol Oncol. 2006;100:70-5.

7. Mc Cormack M, Thomas H. A phase IB study of gemcitabine and concurrent radiotherapy in carcinoma of the cervix. Ann Oncol 2000;11:88.

8. Sirvastava N, Sahani K, Srivastave M. Comparative efficacy of cisplatin vs. gemcitabine as concurrent chemotherapy for untreated locally advanced cervical cancer: A randomized trail. Internet Journal of Medical Update 2007; 2:4-9.

9. Cancer Therapy Evaluation Program. Common Terminology Criteria for Adverse Events, Version 3 DCTD, NCI, NIH, DHHS, 2003. Available from http://ctep.cancer.gov/ 
10. Cox JD, Stetz J, Pajak TF. Toxicity criteria of the Radiation Therapy Oncology Group (RTOG) and the European Organization for Research and Treatment of Cancer (EORTC). Int J Radiat Oncol Biol Phys. 1995;31:1341-6.

11. Eisenhauer EA, Therasse P, Bogaerts J, et al. New response evaluation criteria in solid tumours: revised RECIST guideline (version 1.1). Eur J Cancer. 2009;45:228-47.

12. Rose PG, Bundy BN, Watkins EB, et al. Concurrent cisplatin-based radiotherapy and chemotherapy for locally advanced cervical cancer. N Engl J Med. 1999;340:1144-53.

13. Peters WA $3^{\text {rd }}$, Liu PY, Barrett RJ 2 ${ }^{\text {nd }}$, et al. Concurrent chemotherapy and pelvic radiation therapy compared with pelvic radiation therapy alone as adjuvant therapy after radical surgery in high-risk early-stage cancer of the cervix. J Clin Oncol. 2000;18:1606-13.
14. Hernández $\mathrm{P}$, Olivera $\mathrm{P}$, Dueñas-Gonzalez $\mathrm{A}$, et al. Gemcitabine activity in cervical cancer cell lines. Cancer Chemother Pharmacol. 2001;48:488-92.

15. Chumworathayi B, Suprasert P, Charoenkwan K, et al. Weekly versus three-weekly cisplatin as an adjunct to radiation therapy in high- risk stage I-IIA cervical cancer after surgery; a randomized comparison of treatment compliance. J Med Assoc Thai. 2005; 88:1483-92.

16. Chumworathayi B, Yuenyao P, Tangvorapongchai $\mathrm{V}$, et al. Weekly gemcitabine and cisplatin concurrent with pelvic irradiation for primary therapy of cervical cancer: report of the first seven cases in Thaiwomen. Radiat Med. 2007; 25:474-9.

17. Khalil A, Shamseddine A, Geara F, et al. Concurrent chemo-radiotherapy for locally advanced cervical cancer: A prospective randomized trial comparing cis-platinum vs paclitaxel. J Clin Oncol. (ASCO Annual Meeting Proceedings) 2005; 23:5163. 